\title{
Evaluating the outcome of first episodes of schizophrenia
}

\author{
J.L. VÁZQUEZ-BARQUERO, M.J. CUESTA, M. DE LA VARGA
}

After nearly a century of research into schizophrenia this illness continues to offer a series of challenging questions, among these we have to emphasise the aspects related to its prognosis and outcome. As is well known, the traditional diagnostic formulation of schizophrenia was partially based on views about these concepts. Thus, a central belief associated with the diagnosis of schizophrenia involved ideas about a negative outcome, upon which, for example, the concept of «dementia praecox» was build.

Although the study of schizophrenia prognosis and outcome was originally limited to the clinical variables of Langfelt (1937), and to the analysis of premorbid social adjustment (Kokes et al., 1977), the development by Strauss \& Carpenter (1974) of a Prognostic Scale for Schizophrenia provided the basis for exploring the importance of other outcome variables like, clinical symptoms, hospitalisation, occupational level, and social interactions. The study of these outcome predictors appears to indicate, at least in recent studies, that schizophrenia outcome is not as negative as was originally believed, and that a favourable outcome tends to be associated with good premorbid adjustment, acute onset, and certain psychopathological characteristics (Jonsson \& $\mathrm{Ny}$ man, 1984).

Modern research also shows that, in investigating schizophrenia prognosis and outcome, it is essential to identify different spheres of outcome (symptomatic, social interaction, work performance, so on). These various spheres have different predictors and tend to be affected deferentially by treatment, thus

Indirizzo per la corrispondenza: Professor J.L. Vázquez-Barquero, Unidad de Investigación en Psiquiatría Social de Cantabria, Hospital Universitario «Marqués de Valdecilla», Av. Valdecilla $s / n, 39008$ Santander (Spain).

Fax: (34) 42-202655. suggesting that schizophrenia outcome is a more complex phenomenon than was originally believed (Strauss \& Carpenter, 1977). It has also been demonstrated that, when evaluating these outcome measures, it is necessary to consider whether any short-term improvement also applies to longer range improvement. The appropriateness of this differentiation is apparent, as it has been shown that variables predicting remission of schizophrenia at two years were no longer relevant after 10 years (Vaillant, 1964). The findings of Stanley \& Lindemayer (1987) have to be interpreted in the same direction; they demonstrate that the prognostic and outcome significance of certain indicators is different in the acute than in the chronic stages of the disease.

Until recently research into the prognosis and outcome of schizophrenia was based on the study of «long-term» patients and on designs using «nonoperationalised» diagnostic concepts and «non-representative» samples of patients, thus raising a series of methodological criticisms about the validity of their findings. At present, however, the interest is centred on analysing this issue from the early stages of the disease. The studies designed to meet this objective have been denominated First Episode Studies of Schizophrenia.

In order to guarantee high levels of reliability, it is necessary, when planning these studies, to take into consideration a series of conceptual and methodological problems. The first of these is related to the convenience of specifying what is meant by «First Episode». This concept entails, in the first place, an ordinal component which stresses the need to investigate exclusively the first psychotic experiences. This is mainly justified by the need to control the effects of the confounding variables related to illness evolution. The second component is delineated by the term «Episode», which could be defined as «the minimum period of time in which patients present 
specific symptoms so as to constitute a distinctive category of psychotic disorder" (Keshavan \& Scholer, 1992). To identify a first episode is, however, a difficult task as frequently schizophrenia presents an insidious onset. This is one of the reasons why researchers tend to operationally define "first episode» as the act of establishing "first contact» with services.

The difficulties of implementing these studies are, however, not limited to these aspects, but extend to the process of patient selection, which has to guarantee adequate levels of completeness and representativeness and also a sufficiently large sample size to allow the performing of reliable statistical analysis. These difficulties which are, to a great extent, inherent in the low incidence of schizophrenia, force researchers to extend the sample gathering stage usually over two years, or alternatively to select a multi-centre design. Both strategies tend to introduce systematic errors, based on the often selective composition of small populations, or on the difficulty of achieving adequate levels of inter-rater reliability in the evaluation of patients. Thus, it is recommended that the size of the sampling area should be balanced to prevent the process of patient selection from extending for more than a period of two years, and at the same time to be able to adequately control the case collecting process.

Several strategies could be used for identifying samples of patients in their first episode of schizophrenia. The ideal method would be to conduct a «one-stage» or a «two-stage» prospective community study directed at assessing all "new cases» of schizophrenia. The low incidence of this illness makes these studies almost impracticable. In order to overcome this difficulty «service utilisation samples» of first episodes of schizophrenia have been used, either by investigating hospital admissions or «contacts» with services.

The study of "service utilisation samples" has often been based, for practical reasons, on the use of case registers (Häfner, 1991). With this method, large numbers of patients can be attained with relatively small effort. They have, however, the inconvenience of relying on retrospective clinical evaluations made by different clinicians and thus providing data which are very often of low quality and not comparable. This is why later studies have directly and prospectively investigated schizophrenics who have established contact with services. Most of these studies, have also introduced standardised clinical diagnostic procedures for diagnosing schizophrenia.
This entails the use of operational diagnostic criteria, and of structured psychiatric interviews like the PSE-CATEGO-ID System (Wing et al., 1974; Wing \& Sturt, 1978).

Among these prospective studies, the direct evaluation of «first admissions» has been the strategy most frequently applied (Shepherd et al., 1989; Johnstone et al., 1990; The Scottish Schizophrenia Research Group, 1987; Ring et al., 1991; Ram et al., 1992). Nevertheless, this strategy has the inconvenience of not including schizophrenics who establish contacts with community services. This introduces a clear bias in the process of patient selection, due to the current policy of promoting community psychiatric treatment. Thus, the obvious alternative would be to identify and study "contacts» for a first episode of schizophrenia with any mental health service. This design, which is considered to be the most "cost-effective», has been adopted, for example, in the WHO Ten Country Study (Sartorius et al., 1986; Jablensky et al., 1992), and in the MAP Project Markers and Predictors of Schizophrenia (Iacono \& Beiser, 1992), and in the Cantabria First Episode Schizophrenic Study (Vázquez-Barquero et al., 1994).

The majority of these studies have incorporated, as one of their objectives, the study of schizophrenia prognosis and outcome and also the identification of outcome predictors. A number of them could, however, be criticised for not having introduced adequate strategies into their designs for evaluating the different spheres of outcome. To overcome this deficiency and to provide a more robust basis for establishing relevant indicators of outcome and prognosis, several measures and instruments could be used. Some of these assess positive health, others investigate ill health, and yet another evaluates social functioning. Many of these have separate physical, psychological and social axes, while there are also combined measures, including measures of quality of life. They could be summarised into the following headings:

\section{a) Objective clinical and psychopathological measures}

The majority of studies have evaluated outcome by measuring the clinical, mainly psychopathological, status of patients. For this purpose earlier studies have mainly related on clinical evaluations of patients made by a professional. The tendency now, 
however, is to use reliable structured psychiatric interviews like the PSE-9 (Wing et al., 1974).

In addition, several scales to measure chronic symptoms of schizophrenia have been developed. Among these we have, for example, the Manchester or the K-G-V Scales (Krawiecka et al., 1977), or the Schizophrenia Change Scale (Montgomery et al., 1978). They could, therefore, be considered as a useful alternative for evaluating the clinical components of schizophrenia outcome and prognosis.

\section{b) Objective measures of social functioning}

As Gulbinat (1983) pointed out, when evaluating outcome in schizophrenia, social dimensions of health and illness should be included in addition to other measures of health. These dimensions are incorporated in the scales developed to measure social disability. Examples of these scales are: the Groningen Social Disability Schedule (Wiersma et al., 1990), the Psychiatric Disability Assessment Schedule (WHO, 1988), or the Social Behaviour Schedule (Wykes \& Sturt, 1986).

In addition, generic measures of illness repercussion and quality of life can be applied to evaluate general well being in schizophrenic patients. Examples of these measures are: the Sickness Impact Profile (Bergner et al., 1976); the Quality of Well Being Scale (Patrick et al., 1973), or the Lancashire Quality of Life Profile.

\section{c) Subjective health and social functioning indicators}

It has often been found that there is a disparity between judgements of patients themselves and of professionals about illness repercussions. This emphasises the convenience of taking into account, when evaluating schizophrenia outcome and prognosis, not only the medical view but also the patients view. More so, if we consider that there is now enough evidence to show that self-perceived health status correlates with mortality and recovery rates. In order to account for this, recent studies are incorporating scales into their designs aimed at measuring patients views about their health and psychosocial status. They focus on experiential, and therefore subjective, components of the illness and thus complement the objective evaluation of health made by professionals.

In conclusion, future studies of First Episodes of
Schizophrenia should continue including the analysis of schizophrenia prognosis and outcome as one of their objectives. To this end, strategies should be incorporated into their designs for evaluating the different spheres of outcome, like the ones herein indicated. The relevance of continuing investigating in this field is based on: 1) the need to clarify theoretical issues, as diagnostic concepts about this illness are still partially based on assumptions about its outcome; and 2) the need to provide a solid base upon which to develop therapeutic and preventive programs.

\section{REFERENCES}

Bergner M., Bobbit R.A. \& Pollard W.E. (1976). The sickness impact profile: validation of a health status measure. Medical Care 14, 157-179.

Gulbinat W. (1983). Mental health problem assessment and information support direction of the WHO's work. World Health Statistics Quarterly 36, 224-233.

Häfner H. (1991). New perspectives in the epidemiology of schizophrenia. In Search for the Causes of Schizophrenia, Vol. 2. (ed. H. Häfner and W.F. Gattaz), pp. 408-431. Springer: Berlin.

Iacono W. \& Beiser M. (1992). Where are the women in FirstEpisode Studies of Schizophrenia? Schizophrenia Bulletin 3, 471-480.

Jablensky A, Sartorius N., Ernberg G., Anker M., Korten A., Cooper J.E., Day R. \& Bertel A. (1992). Schizophrenia: manifestations, incidence and course in different cultures. A World Health Organization Ten-Country Study. Psychological Medicine, Supplementum No. 20, pp. 3-97.

Johnstone E.C., MacMillan J.F., Frith C.D., Benn D.K. \& Crow T.J. (1990). Further investigation of the predictors of outcome following first schizophrenic episodes. British Journal of Psychiatry 157, 182-189.

Jonsson H. \& Nyman A.K. (1984). Prediction of outcome in schizophrenia. Acta Psychiatrica Scandinavica 69, 274-279.

Keshavan M.S. \& Schooler N.R. (1992). First-episode studies in schizophrenia: criteria and characterization. Schizophrenia Bulletin 18, 491-513.

Kokes R.F., Strauss J.S. \& Klormant R. (1977). Part II. Measuring premorbid adjustment: the instruments and their development. Schizophrenia Bulletin 3, 186-213.

Krawiecka M., Goldberg D.P. \& Vaughan M. (1977). A standardized psychiatric assessment scale for rating chronic psychotic patients. Acta Psychiatrica Scandinavica 55, 299-308.

Langfelt G. (1937). The prognosis of schizophrenia and the factors influencing the course of the disease. Acta Psychiatrica Neurologica Scandinavica, Supplementum No. 13.

Montgomery S.A., Taylor P. \& Montgomery D. (1978). Development of a schizophrenia scale sensitive to change. Neuropharmacology 17, 1061-1063.

Patrick D.L., Bush J.W. \& Chen M. (1973). Methods of measuring levels of well-being for a health status index. Health Service Research 8, 228-231. 


\section{J.L. Vázquez-Barquero}

Ram R., Bromet E.J., Eaton W.W., Pato C. \& Schwartz J.E. (1992). The natural course of schizophrenia: a review of firstadmission studies. Schizophrenia Bulletin 18, 185-207.

Ring N., Tantam D., Montague. L. Newby D., Black D. \& Morris J. (1991). Gender differences in the incidence of definite schizophrenia and atypical psychosis: focus on negative symptoms of schizophrenia. Acta Psychiatrica Scandinavica 84, 489-496.

Sartorius N., Jablensky A., Korten A., Ernberg G., Anker M., Cooper J.E. \& Day R. (1986). Early manifestations and firstcontact incidence of schizophrenia in different cultures. Psychological Medicine 16, 909-928.

The Scottish Schizophrenia Research Group (1987). The Scottish first episode schizophrenia study. I. Patient identification and categorisation. British Journal of Psychiatry 150, 331-333.

Shepherd M., Watt D., Falloon I. \& Smeeton N. (1989). The natural history of schizophrenia: a five-year follow-up study of outcome and prediction in a representative sample of schizophrenics. Psychological Medicine, Supplementum No.15, pp. 1-46.

Stanley R.K. \& Lindemayer J.P. (1987). Outcome predictors in acute schizophrenia. Prospective significance of background and clinical dimensions. The Journal of Nervous and Mental Disease 175, 152-160.

Strauss J.S. \& Carpenter W.T. (1974). Prediction of outcome in schizophrenia: II. Relationships between predictor and out- come variables. Archives of General Psychiatry 31, 37-42.

Strauss J.S. \& Carpenter W.T. (1977) Prediction of outcome in schizophrenia. III. Five-year outcome and its predictors. Archives of General Psychiatry 34, 159-163.

Vaillant G. (1964). Prospective prediction of schizophrenic remission. Archives of General Psychiatry 11, 509-518.

Vázquez-Barquero J.L., Cuesta Nuñez N.J., Varga M., Herrera Castanedo S., Gaite L. \& Arenal A. (1994). The Cantabria first episode schizophrenia study: a summary of general findings. Acta Psychiatrica Scandinavica (accepted for publication).

Wiersma D., De Jong A. \& Kraaijkamp H.J.M. (1990). The Groningen Social Disabilities Schedule. 2nd Version. University of Groningen. Department of Social Psychiatry: Groningen.

Wing J.K. \& Sturt E. (1978). The PSE-ID-CATEGO System: a Supplementary Manual. Institute of Psychiatry: London.

Wing J., Cooper J.E. \& Sartorius N. (1974). Measurement and Classification of Psychiatric Symptoms. Cambridge University Press: Cambridge.

World Health Organization (1988). Psychiatric Disability Schedule (WHO/DAS). WHO: Geneva.

Wykes T. \& Sturt E. (1986). The measurement of social behaviour in psychiatric patients: an assessment of the reliability and validity of the SBS schedule. British Journal of Psychiatry 148, 1-11. 